\title{
Educating and training biomedical researchers in biophotonics and advanced light microscopy methods
}

\section{Thomas Abraham}

Thomas Abraham, "Educating and training biomedical researchers in biophotonics and advanced light microscopy methods," Proc. SPIE 11143, Fifteenth Conference on Education and Training in Optics and Photonics: ETOP 2019, 1114326 (2 July 2019); doi: 10.1117/12.2532012 Photonics: ETOP 2019, 2019, Quebec City, Quebec, Canada 


\title{
Educating and Training Biomedical Researchers in Bio- photonics and Advanced Light Microscopy Methods
}

\author{
Thomas Abraham, $\mathrm{PhD}^{1,2}$ \\ ${ }^{1}$ Department of Neural \& Behavioral Sciences, ${ }^{2}$ Microscopy Imaging Core Lab, \\ Pennsylvania State University College of Medicine, Hershey, Pennsylvania 17033
}

Corresponding Author: tua17@psu.edu or tabraham1@pennstatehealth.psu.edu

\begin{abstract}
Biological research is complex these days because of technological challenges associated with biophotonics and advanced light microscopic methods. Core labs with appropriate advanced optical technological tools along with proper education and training can be an integral part and play a vital role in achieving complex biomedical research objectives. Advanced light microscopy tools can meet all specific needs but are so complex to learn with regard to obtaining optimal imaging datasets. Therefore, a solid educational and training program are essential. Based on researcher's specific needs, there can be two major types of training and education, a general training for all the researchers and a specific individualized training for researchers who want to explore complex experiments.
\end{abstract}

KEYWORDS: Education and Training; Biomedical Researchers; Bio-photonics; Advanced Light Microscopy Methods; Multidimensional high definition imaging; General training; Specific individualized training; Complex quantifications

\section{INTRODUCTION}

Biological research is complex these days because of technological challenges associated with bio-photonics and advanced light microscopic methods. Core labs with appropriate advanced optical technological tools along with proper education and training can be an integral part and play a vital role in achieving complex biomedical research objectives. In subcellular level imaging, a biomedical researcher is interested in various aspects. For instance, the spatial resolution which facilitates direct visualization of sub cellular structures or structural changes, the time resolution which enables visualization of sub cellular events or physiological changes in real time, the spectral resolution which facilitates identification of biomarkers/ sub cellular structures, and quantitative measures which enable quantitative comparison among different groups.

Advanced light microscopy tools can meet all such specific needs but are so complex to learn with regard to obtaining optimal imaging datasets. With regard to optics and image formation, a biomedical researcher is primarily interested in spatial resolution, which is a measure of how fine details can be detected and the images free of distortions resulting from chromatic (Color shift) and spherical aberrations. The way to improve the resolution is collecting higher orders of diffracted light, so one has to use high numerical aperture (NA) lens with appropriate working distance and aberration corrections. In addition, one has to use appropriate immersion media matching refractive index with mounting media or buffers in live cell imaging as the refractive index mismatch in optical path amplifies image distortions or aberrations. Compromise of various optical parameters including objective lens selection, immersion media, laser source, laser power among others are needed for obtaining optimal 3D image datasets [1-3]. 
Control software programs which drive these instruments are equally complex but offer unlimited options and functions. With regard to image acquisition, a biomedical researcher is interested in best possible images which are free of both background and noise utilizing the resolving power of the microscope. Therefore the appropriate steps in image registration and proper sampling (statistics) such as appropriate gain/ offset levels (in the case of PMT detectors), line or frame averaging for high signal to noise ratio, appropriate pixel size for Nyquist sampling, appropriate pixel dwell time are needed for optimal results [1-3]. Compromise of these imaging acquisition parameters are needed for generating optimal imaging datasets. Nyquist sampling is particularly important in post image processing operations such as deconvolution.

The fluorescence chemical properties such as excitation, emission and decay time can also be exploited in multicolor 3D imaging experimental settings to differentiate various fluorescently labelled subcellular objects [1-3]. In addition, high end microscopes such as multiphoton integrated with ultra-short femtosecond IR laser pulses can be operated in multiple modalities [4, 5]. For instance, such femtosecond IR laser pulses can induce harmonic generation signals from non-centrosymmetric materials such fibrillar collagens, myosin and microtubules without the need of any prior exogenous labelling, as well as can induce endogenous fluorescence from elastin, macrophages, reduced $\mathrm{NAD}(\mathrm{P}) \mathrm{H}$ or oxidized FAD [4-6]. Also, the harmonic generation signals can be collected in backward and forward directions simultaneously which enable one to differentiate fibrillar collagen phenotypes or mature versus immature fibrillar collagens [7].

There are also dedicated high end image data processing software programs with various quantification approaches which include, for instance, IMARIS, VOLOCITY and HUYGENS image processing work stations. These high-end programs facilitate $3 \mathrm{D}$ and $4 \mathrm{D}$ reconstructions to visualize and analyze multiple color images, renderings, deconvolution, segmentation, fluorescence intensity measurements, complex cell tracking measurements, voxel counting, batch processing, drug bio-distribution measurements, accurate co-localization analysis and many other complex image processing as well as statistical calculations depending upon researcher's needs. For instance, image processing software such as VOLOCITY enable us to develop image processing protocols and process hundreds of 3D or 4D images and compile the data in multiple ways [8]. In addition programming platform like MATLAB can be utilized to perform Fourier transformation of image data from spatial to frequency domain which enables us to compute orientation properties of fibrillar collagens [8].

Considering all these complexities, a solid educational and training program is essential to educate and train biomedical researchers in relevant areas particularly in bio-photonics and advanced light microscopy methods. The objective here is to develop educational and training methodologies for biomedical researchers. Based on researcher's specific needs, there can be two major types of training and education programs, a general training for all the researchers and a specific individualized training for researchers who want to explore complex multidimensional 3D or 4D experiments. This is particularly relevant when the imaging projects involve both multidimensional high-resolution imaging and subsequent complex quantifications.

\section{EDUCATION \& TRAINING APPROACHES}

Because of all these complexities in modern day biomedical research, a solid training core training and education program which includes teaching materials, evaluation method such as quiz to test researcher's knowledge are essential. Before we train researchers in bio-photonics, first we collect specific details regarding their proposed imaging studies including biosafety aspects. Based on their inputs, we arrange a short one-to-one meeting to know more about researcher's 
specific need. Following the meeting, we categorize them into two user groups such as basic user and advanced user groups.

Basic training will enable researchers to achieve imaging goals such as visualizing multiple objects in fixed cells or tissues with multiple colors in 3D, spectral assessments to confirm the specificity of a fluorescent probes among others. Text books for instance [1-3], Microscopy education websites, Vendors websites, Instrument manuals, etc., are good sources for developing basic training and education materials.

Certain research projects have very demanding specific needs which cannot be met with basic training. Therefore, we need a specific individualized training for advanced user group. This is particularly relevant when the imaging projects involve both multidimensional high-resolution live cell/ deep tissue ex vivo/ in vivo/ intravital microscopic imaging and subsequent complex quantifications such as real time physiological events, object tracking, drug bio-distributions, DNA ploidy measurements, harmonic generation and multiphoton imaging among others. Core personnel may not be able to achieve these objectives their own. Strong collaborative efforts among researchers and core personnel are needed to achieve these specific goals.

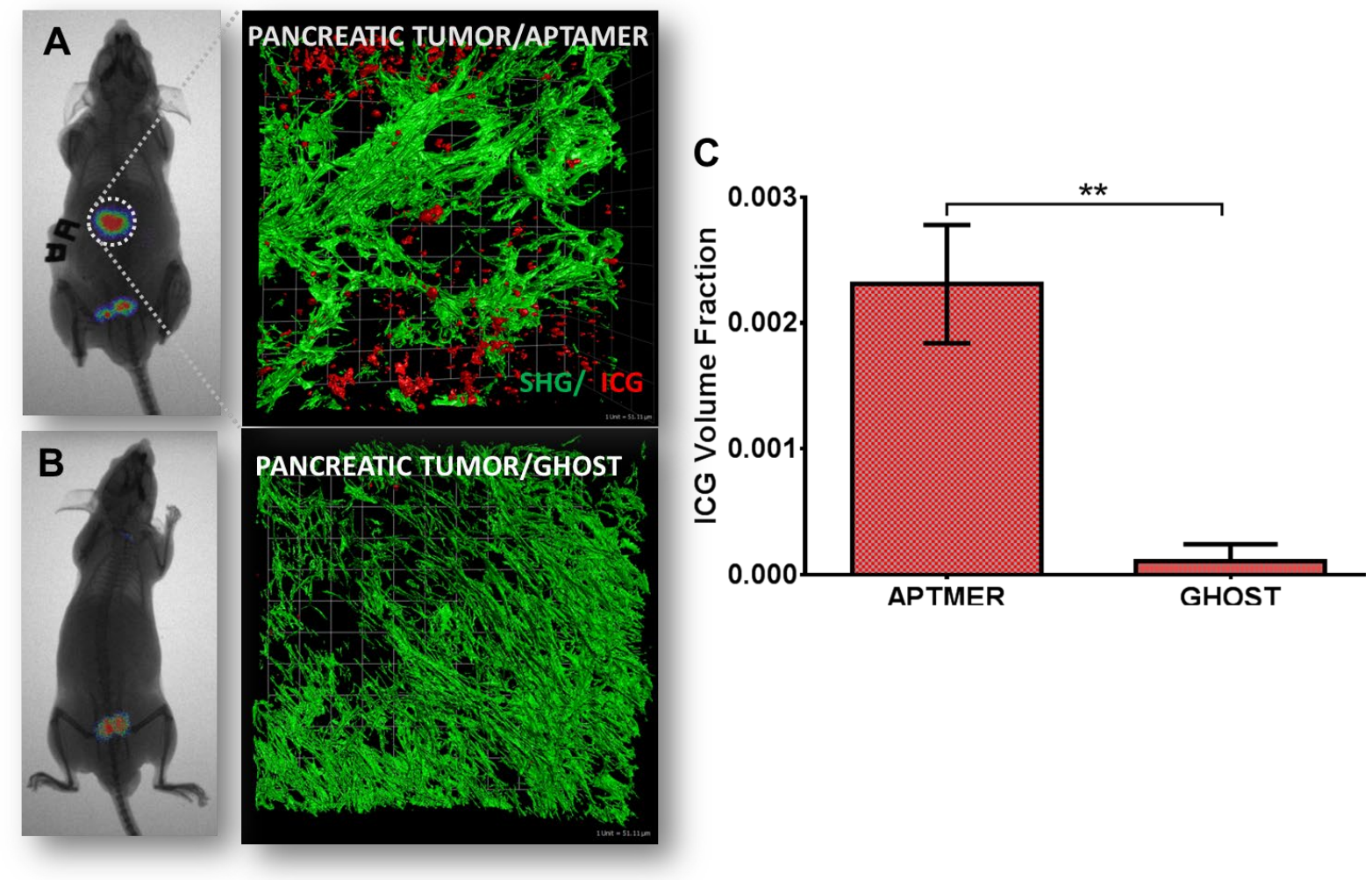

Figure 1: An example of research output of specific individualized training using multiphoton and harmonic generation microscopy methods. Ex vivo imaging of whole pancreatic tumor cross sections $(\sim 300 \mu \mathrm{m})$ using multiphoton microscopy showing the localization of ICG loaded nanoparticles (Red) relative to fibrillar collagen regions of the tumor (Green). (A) Mouse bearing tumor injected with targeted aptamer conjugated nanoparticles shows relatively high ICG signal throughout the tumor section; (B) Mouse bearing tumor injected with nanoparticles without ICG (ghost particles) shows no ICG signal; (C) Computed ICG volume fractions between targeted versus ghost (empty ICG) particles (Adapted from [9]). 
There are steps in specific individualized training for advanced user group. First, a clear understanding of biological problem and in-depth discussion with biologists to understand what they are after. Published works in the similar areas may be useful for this purpose. Second, identify right instrument and software. For instance, if the project needs high speed imaging, select a microscope with high speed scanning capability. If the project requires high resolution imaging, select a microscope with high resolving power and ignore the speed capability. Third, select appropriate cell component markers with minimum cross talks and cross excitations. This is particularly relevant in live cell imaging. Forth, set appropriate data acquisition parameters. For instance, if the objective is to visualize and quantify the bio-distribution of drug in ex-vivo highly fibrotic whole pancreatic tumor tissue with structural precision [9], select multiphoton and harmonic generation 3D Imaging platform, select objective lens with high resolving power and long working distance, select dyes preferably with red emissions for labeling, employ sufficient number of pixels (Nyquist sampling), use appropriate pixel dwell time and frame averaging, to generate high definition 3D deep tissue images. If the subject is quantitative multicolor live cell imaging, where for instance the objective is to visualize and interactions of a GFP expressed protein with mitochondria in transfected mammalian cells in real time [10], select microscope with zoom-in capability, acquire both channels simultaneously, use markers with minimum cross talks and cross excitations, employ sufficient number of frame per second, use appropriate pixel size and pixel dwell time, and appropriate zoom level and low laser power. Many of these parameters are conflicting, therefore compromise among these parameters are important for optimal results. Fourth, use positive control if available and acquire data using negative control as well if applicable. Last, process the data using the same computational algorithms, start with positive control, apply the same algorithm to all the samples and use statistical tools to test significance.

\section{GENERAL SUMMARY}

In summary, because of technological complexities involved in modern day biomedical research, education and training methods are very essential for achieving complex biomedical research goals. Collecting experimental info from researchers are important as initial steps for the development of training programs. Based on user's specific needs, there can be two major types of training programs; training for basic users and specific individualized training for advanced users. Team work among biologists and biophysicists are vital for the development of result oriented specific individualized training programs.

\section{REFERENCES}

[1] G. Cox, [Optical imaging techniques in cell biology] CRC/Taylor \& Francis, Boca Raton(2007).

[2] A. R. Hibbs, [Confocal microscopy for biologists] Kluwer Academic/Plenum Publishers, New York(2004).

[3] J. B. Pawley, [Handbook of biological confocal microscopy] Springer, New York(2006).

[4] W. R. Zipfel, R. M. Williams, R. Christie et al., "Live tissue intrinsic emission microscopy using multiphoton-excited native fluorescence and second harmonic generation," Proc Natl Acad Sci U S A, 100(12), 7075-80 (2003).

[5] P. Campagnola, "Second harmonic generation imaging microscopy: applications to diseases diagnostics," Anal Chem, 83(9), 3224-31 (2011).

[6] T. Abraham, J. A. Hirota, S. Wadsworth et al., "Minimally invasive multiphoton and harmonic generation imaging of extracellular matrix structures in lung airway and related diseases," Pulm Pharmacol Ther, 24(5), 487-96 (2011). 
[7] M. Suzuki, D. Kayra, W. M. Elliott et al., "Second Harmonic Generation Microscopy Differentiates Collagen Type I and Type III in COPD," Multiphoton Microscopy in the Biomedical Sciences Xii, 8226, (2012).

[8] T. Abraham, D. Kayra, B. McManus et al., "Quantitative assessment of forward and backward second harmonic three dimensional images of collagen Type I matrix remodeling in a stimulated cellular environment," J Struct Biol, (2012).

[9] T. Abraham, G. A. Clawson, S. S. Linton et al., "Bio-distribution of Near Infrared Imaging Agent Loaded Targeted Drug Nanoparticle Carriers in Highly Fibrotic Pancreatic Tumor Determined Using Multiphoton and Harmonic Generation Imaging," Imaging, Manipulation, and Analysis of Biomolecules, Cells, and Tissues Xvi, 10497, (2018).

[10] P. Chadha, A. Sarfo, D. A. Zhang et al., "Domain Interaction Studies of Herpes Simplex Virus 1 Tegument Protein UL16 Reveal Its Interaction with Mitochondria," Journal of Virology, 91(2), (2017). 